\title{
Mobile Learning na Sala de Aula Presencial: Repositório Semântico de Objetos de Aprendizagem para Dispositivos Móveis
}

Fabio Teixeira Franciscato, PPGI/UFSM, fabiofranciscato@yahoo.com.br Roseclea Duarte Medina, PPGI/UFSM, roseclea.medina@ gmail.com Patricia Mozzaquatro, PPGI/UFSM patriciamozzaquatro@gmail.com Patric Ribeiro, PPGI/UFSM, patricribeiro@uabrestingaseca.com.br

\section{Resumo}

Objetos de Aprendizagem (OA) são abordagens pedagógicas de grande valia no ensino, facilitando a aprendizagem de conteúdos de forma animada e interativa. Com o advento da Mobile Learning, OA estão sendo criados ou adaptados para execução em dispositivos móveis. Diante das características da computação Móvel, este artigo propõe um Repositório semântico de Objetos de Aprendizagem para dispositivos móveis que, automaticamente, pesquisa por OA em função do contexto do aprendiz, sem sua interferência, levando em consideração as características do dispositivo móvel a ser utilizado.

Palavras-chaves: m-learning, computação móvel, web semântica, ontologias

\section{Mobile Learning at the Classroom: Semantic Repository of Learning Objects for Mobile Devices}

\begin{abstract}
Learning Objects are pedagogical approaches of great value in teaching, facilitating learning content in a lively and interactive style. With the advent of Mobile Learning, many OA are being created or adapted to run on mobile devices. Given the characteristics of mobile computing, this paper proposes a semantic repository of learning objects for mobile devices that will automatically search for objects in function of the context of the user, without his interference, taking into consideration the characteristics of the mobile device used.
\end{abstract}

Keywords: m-learning, mobile computing, semantic web, ontologies

\section{Introdução}

O cenário computacional, em constante evolução, se depara diante da evolução, onde não mais o usuário se adapta à tecnologia, mas sim, as tecnologias se adaptam em função do usuário. Isto é, em um ambiente de Computação Móvel (Weiser, 1991), o contexto do usuário servirá de referência para os sistemas computacionais.

A Computação Móvel tem por característica funcionar de modo invisível ao usuário. Todavia, estará reagindo às mudanças de contexto, de modo a disponibilizar somente informações relevantes ao usuário em função do ambiente em que se encontra (Augustin, 2004). A computação móvel, quando em um contexto de aprendizagem, caracteriza um novo paradigma na educação, a Mobile Learning (M-Learning)

A M-Learning é uma extensão do E-Learning e é praticada através de dispositivos móveis, como celulares, smartphones, permitindo assim uma maior condição de acesso a recursos pedagógicos, independente de tempo e lugar (Starr, 2007). Muitos sistemas de ensino estão sendo adaptados para execução e visualização em dispositivos móveis. Objetos de Aprendizagem também vêm ganhando espaço neste cenário. 
A combinação de características da M-Learning (personalizada, centrada no aprendiz, colaborativa) com característica de Objetos de Aprendizagem (conteúdo digital reutilizável, granularidade) pode produzir uma poderosa ferramenta para suportar aprendizagem a qualquer hora e lugar (Castillo, 2008).

Em sala de aula presencial, a utilização de Objetos de Aprendizagem pode ser um bom recurso pedagógico para facilitar o processo de ensino/aprendizagem de um conteúdo, facilitando a construção do conhecimento dos alunos (Tavares, 2007). Porém, nas escolas, a execução de OA se limita somente aos desktops, trazendo uma variedade de dificuldades, como falta de computadores para todos os alunos, indisponibilidade, ou até mesmo, prejuízo de tempo com deslocamento dos alunos até laboratórios de informática.

Diante desses problemas/dificuldades e levando em consideração que uma grande porcentagem dos alunos dispõe de dispositivos móveis em sala de aula, este artigo apresenta uma proposta de Repositório de Objetos de Aprendizagem para dispositivos móveis que utiliza características da Web Semântica e da Computação Móvel.

O Repositório proposto identificará, sem a interferência do aluno, a partir de prévia identificação do conteúdo pelo professor, todos os OA disponíveis para o conteúdo proposto e que possam ser executados no dispositivo móvel do aluno. Para isso, foi utilizada a ontologia de Objetos de Aprendizagem proposta por Franciscato (2009), padrões da Web Semântica (W3C, 2001) e as características da Computação Móvel.

\section{Computação Móvel no contexto de Ensino/Aprendizagem}

Os avanços da tecnologia centrados na mobilidade vêm transformando a maneira de como o usuário "enxerga" a tecnologia. Interfaces inteligentes, recursos multimídia, comunicação sem fio, altas velocidades no acesso aos dados da web. Todas essas tecnologias juntas em um único dispositivo estão ocasionando um amplo crescimento do uso destas tecnologias por usuários de todo o mundo.

Segundo a União Internacional de Telecomunicação (ITU, 2009) durante o ano de 2008 foi alcançada a marca de 4.1 bilhões de dispositivos móveis no mundo, Figura 1. Reforçando a magnitude alcançada pela Computação Móvel.

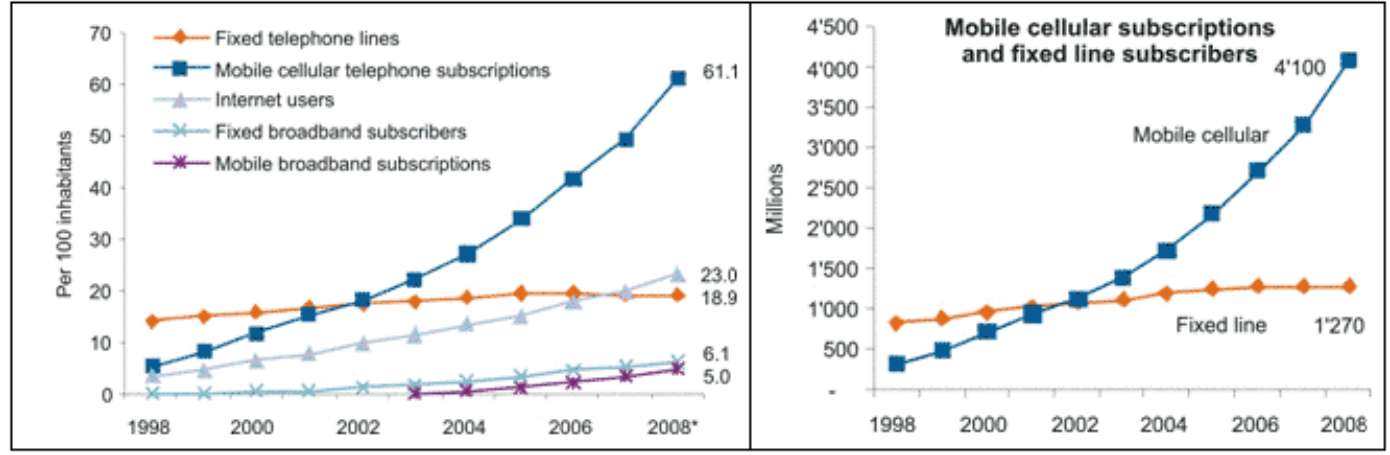

Figura 1 - Crescimento do número de dispositivos móveis no mundo

Diante das características e da dimensão que a Computação Móvel vem atingindo, é fato que, o uso de dispositivos móveis está alcançando todos os tipos de 
usuários para todos os tipos de utilização. Sua utilização, em um cenário educacional, é caracterizada como Mobile Learning.

Tecnologias móveis na educação podem proporcionar benefícios tanto aos alunos quanto aos professores. Aos alunos é proporcionada uma maior flexibilidade na aprendizagem, sendo que o material está acessível através de seus dispositivos móveis, permitindo-lhes aprender como e quando for necessário, não importando onde estejam, mesmo que em movimento. Aos educadores é fornecido um novo meio de disponibilização do material pedagógico, como também um novo meio de interação com o aluno (Siau, 2008).

Considerando a construção de soluções de aprendizagem com mobilidade, é necessário que estas devam priorizar os critérios de usabilidade, acessibilidade, mobilidade, colaboração/cooperação (Schlemmer, 2007). Os OA empregam essas características e podem ser acessados, dependendo de alguns fatores como hardware e software, por dispositivos móveis.

\section{Objetos de Aprendizagem para Dispositivos móveis}

Objetos de Aprendizagem são "quaisquer recursos digitais que possam ser utilizados/reutilizados para o suporte ao ensino" (Wiley, 2002). Como recurso digital entende-se, nessa situação, simulações, animações, vídeos, fotos.

A grande vantagem dos OA é a sua distribuição na internet, o que possibilita o acesso por qualquer pessoa a qualquer hora e, com o advento da M-Learning, acesso a partir de qualquer local.

Quando se trata de Objetos de Aprendizagem para dispositivos móveis, já existem várias publicações na área como (Oliveira, 2007), (Cruz, 2008), (Toledano, 2006), (Franciscato, 2008) entre outros, os quais expõem um novo paradigma para Objetos de Aprendizagem: a sua utilização não mais associada a desktops e sim a dispositivos móveis, como celulares e Smartphones. Alguns exemplos das publicações citadas, na Figura 2.
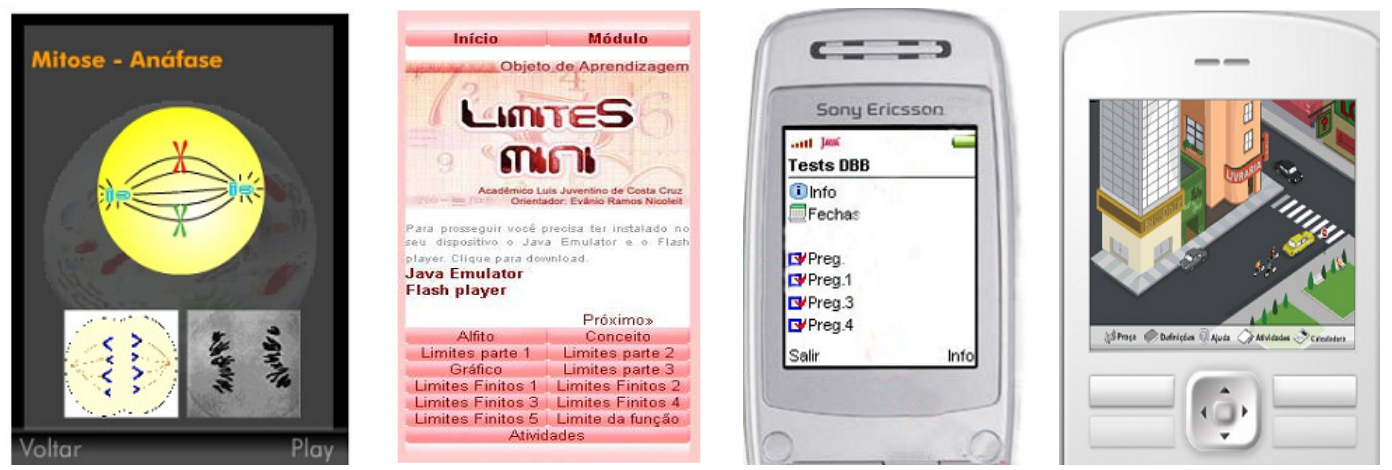

Figura 2. Exemplos de Objetos de Aprendizagem para dispositivos móveis

Uma dificuldade encontrada na execução de Objetos de Aprendizagem para dispositivos móveis refere-se à incompatibilidade em relação a Sistemas Operacionais e configuração de Hardware para cada fabricante. Isto, pois, os OA podem estar programados em diversas linguagens de programação, como Flash, J2me, Html, Android, Iphone, entre outros, e depender de velocidade de processamento ou quantidade de memória ram, os quais podem estar indisponíveis em alguns aparelhos. 
O repositório proposto neste artigo filtrará automaticamente os Objetos de Aprendizagem aptos a serem executados no dispositivo móvel do aluno, como também filtrará os OA em relação ao contexto da aula (conteúdo) em que se encontra o aluno.

\section{Ontologia e Semântica no Repositório de Objetos de Aprendizagem para Dispositivos Móveis.}

Ontologias foram recentemente adotadas pelas comunidades de inteligência artificial (IA) e gestão de conhecimento para se referir aos conceitos e termos que podem ser usados para descrever alguma área do conhecimento ou construir uma representação desses (Guimarães, 2002), (Araujo, 2003).

Seus objetivos são de possibilitar a formalização, reutilização e o compartilhamento do conhecimento. Esses objetivos, que despertaram o interesse dos pesquisadores de IA há vários anos, ganharam maior atenção com o advento da Web Semântica (Neto, 2006).

A ontologia proposta neste projeto é uma adaptação/aperfeiçoamento da ontologia encontrada em (Araujo, 2004) e seu principal objetivo é possibilitar uma representação semântica dos OA para dispositivos móveis, a fim de que estes Objetos possam ser estruturados, de forma que o sistema possa encontrar resultados condizentes com o contexto do aluno em sala de aula.

Como apresentado na Figura 3, a ontologia descreve um Objeto de Aprendizagem como parte de um Tópico, este parte de um módulo e o módulo parte de uma disciplina. Exemplificando: Um objeto está incluído na disciplina de "Biologia", no módulo "Genética" e no tópico "Dominância e recessividade". Este objeto é criado por uma equipe e é originado de uma universidade. Este objeto tem propriedades, como a configuração necessária de hardware e software para ser executado e pode ser executado em um dispositivo móvel.

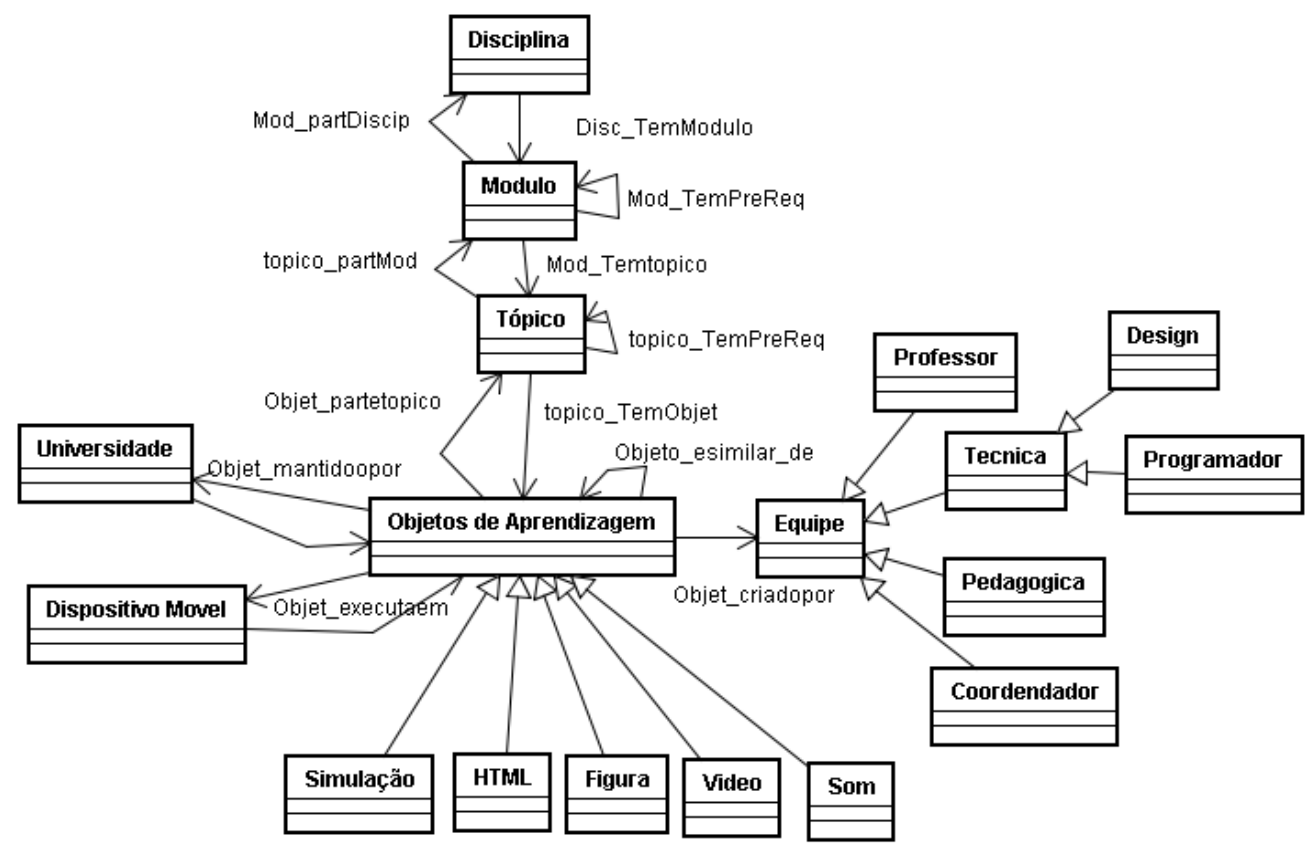

Figura 3. Ontologia para Objetos de Aprendizagem para dispositivos móveis 
Com a aplicação desta ontologia, um objeto de aprendizagem estará relacionado a módulos e tópicos de uma disciplina, por este motivo, este não estará "solto" dentro de um repositório. É possível saber, claramente, o assunto tratado no objeto; assim como saber quantos e quais OA tratam do mesmo assunto, navegar entre OA que tratem de assuntos anteriores (pré-requisitos) ou OA que dêem continuidade ao assunto tratado. Pode-se saber também quais OA foram feitos por uma mesma equipe, ou quais Objetos foram produzidos por uma única instituição de ensino, e ainda em quais dispositivos móveis ou configurações de hardware e software que o objeto está apto a ser executado.

Para evitar que Objetos de Aprendizagem associados ao repositório proposto estejam identificados por simples palavras chaves, o que impossibilitaria qualquer relação semântica entre os mesmos, tornando o sistema incapaz de relacionar e identificar significados sem a interferência humana, o repositório está baseado na estrutura da Web Semântica.

Diante do crescimento sem limites da Web em relação à quantidade e complexidade de informações, torna-se imprescindível a utilização de tecnologias capazes de manipular as informações de forma automática. No atual estado da Web, o acesso, interpretação e extração das informações são deixadas a cargo dos usuários.

Segundo Berners-Lee (2001): “A Web Semântica não é uma Web separada, mas uma extensão da atual, na qual a informação é utilizada com significado bem definido, aumentando a capacidade dos computadores para trabalharem em cooperação com as pessoas". A Web Semântica proporcionará uma estrutura que viabilize a compreensão e gerenciamento dos conteúdos armazenados na Web, independendo da maneira de apresentação dos mesmos, podendo ser som, texto, imagens, animações (Hendler, 2002), (Bizzarro, 2006) (Ferreira, 2006).

\section{Utilização do Repositório Semântico de OA para Dispositivos Móveis em sala de aula presencial.}

A utilização de Objetos de Aprendizagem como recurso pedagógico adicional a uma aula presencial pode ser de grande valia no processo de ensino/aprendizagem. Porém, diante de alguns problemas, a utilização de OA pode ser deixada de lado.

A primeira categoria de problemas se refere-se à falta de estrutura das escolas em relação aos equipamentos necessários para execução de um Objeto de Aprendizagem, isto é, a grande maioria das escolas não disponibiliza computadores aos alunos em sala de aula. O que então é solucionado com a utilização de laboratórios de informática, o que pode trazer mais problemas como, o tempo perdido com $o$ deslocamento dos alunos até o laboratório e a necessidade do professor em manter os alunos no laboratório por todo um período de aula ou mais, pelo motivo de poucos minutos de acesso e utilização de um OA. Estes, para esta proposta, são os principais motivos para a utilização da Computação Móvel.

A segunda categoria de problemas se refere à limitação dos Repositórios de OA, como por exemplo, o Rived (Rived, 2004), e dois dos maiores do mundo, o Merlot (Merlot, 1997) e o Wisc (Wisc, 2000) que disponibilizam apenas buscas por palavras chaves e alguns poucos tipos de filtros para a pesquisa por OA de aprendizagem, o que pode dificultar, ou até levar a desistência de um professor em relação à utilização de objetos de aprendizagem em sala de aula. Estes são os principais motivos da aplicação dos padrões da Web Semântica e utilização de ontologias para expressar o domínio de OA no repositório. 


\subsection{Computação Móvel na sala de aula}

A utilização da computação Móvel aperfeiçoa a maneira de como o aluno em sala de aula presencial acessa Objetos de Aprendizagem. $\mathrm{O}$ aluno não necessitará fazer qualquer tipo de pesquisa/busca por OA, o sistema apresentará automaticamente os Objetos de Aprendizagem que se encaixem no contexto da disciplina e conteúdo abordados em aula.

Para isso, como apresenta a Figura 4, será necessário um Servidor, que vai manter os dados do Repositório de Objetos de Aprendizagem, e um Switch, que vai conectar o Servidor a rede se fio (Wi-Fi), permitindo, assim, o acesso dos alunos ao repositório.

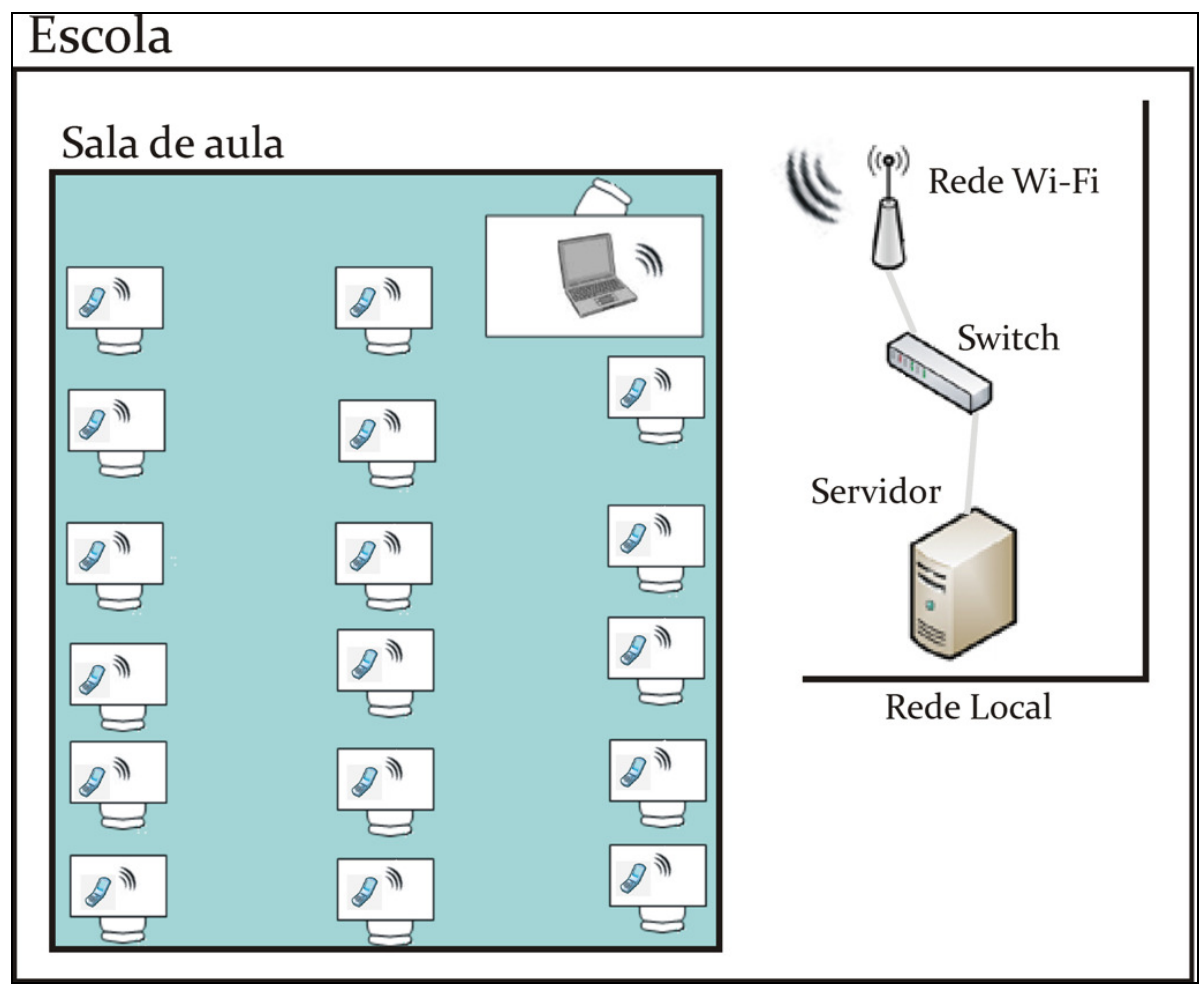

Figure 4. Sala de aula com utilização de tecnologias móveis

Para tal cenário Móvel, será necessário que os dispositivos móveis dos alunos, no mínino, possam acessar redes Wi-Fi, evitando qualquer tipo de custo com operadoras de telefonia. Para os professores será necessário algum meio de acesso ao repositório, no caso do apresentado na Figura 4, um notebook com acesso a rede sem fio, o qual vai permitir que o professor possa fazer mudanças de conteúdo a qualquer momento da aula, alterando o contexto do aluno.

\subsection{Ontologia e Web semântica no repositório para Objetos de Aprendizagem}

A Computação Móvel tem por objetivo ser transparente ao usuário, isto é, o aluno terá ao alcance de suas mãos os OA específicos para aquela situação do contexto da sala de aula em que se encontra, sem sua interferência direta. A partir disso, o quem vem em mente é, como um sistema poderia fazer esta seleção de OA sem a interferência do usuário e, ainda por cima, sabendo que computadores não têm a capacidade de 
interpretar informações? Isso remete ao uso de Ontologias e a adoção dos padrões da Web Semântica.

A Ontologia tem como característica a representação do conhecimento sobre um domínio, neste caso, o conhecimento sobre Objetos de Aprendizagem para dispositivos móveis. A utilização da ontologia juntamente aos padrões da Web Semântica permitiu ao sistema a atribuição e conexão de significados aos Objetos de Aprendizagem e suas características. Abaixo, no item 5.3, é apresentado a implementação dessa abordagem.

\subsection{Implementação}

Para fins de implementação computacional, a ontologia abstrata da Figura 3 necessitou ser codificada, para isso, a mesma foi editada no editor de ontologias Protégé (Protégé, 1997), o qual permitiu a geração de classes, tipos, relacionamentos e cardinalidades, como mostra a Figura 5, como também, permitiu a exportação dessas informações para a linguagem de marcação semântica Web Ontology Language (OWL).
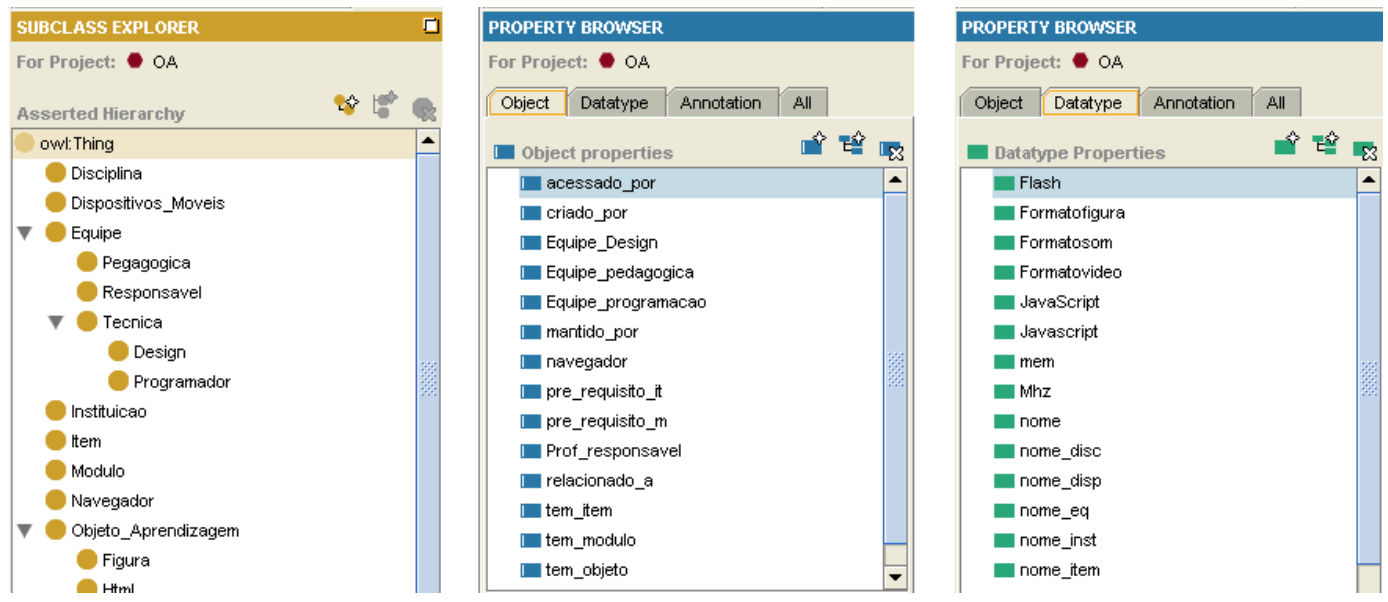

Figura 5. Ontologia descrita no editor Protégé

A linguagem OWL é baseada nas tecnologias da Web Semântica XML, RDF e RDF Schema. A linguagem de marcação XML permitiu adicionar estruturas de forma concisa e universal aos documentos. Todavia, não explicitou o que tais estruturas significavam (semântica). Então, através do RDF e RDF Schema estes significados puderam ser representados e relacionados, o que expressou o conhecimento codificado no formato de triplas (sujeito, predicado, objeto), isto é, o conhecimento foi decomposto em pedaços para melhor estruturar as informações e permitir a inclusão da semântica. $\mathrm{O}$ sujeito representa o conceito (classes), o predicado são as propriedades e o objeto são os valores.

Para manipular os dados foi utilizada a linguagem PHP e a biblioteca RDF API for PHP (RAP), que é um conjunto de ferramentas de código aberto que tem por objetivo manipular dados baseados na estrutura da Web Semântica (Rap, 2002).

Para recuperação dos dados armazenados no repositório foi utilizada a linguagem de consultas, recomendada pela W3C e suportada pelo RAP, SPARQL (SPARQL Query Language for $R D F$ ), que pode realizar consultas sobre ontologias descritas em RDF (Sparql, 2008), o caso deste projeto.

\subsection{Testes}


Os testes foram feitos em duas etapas, primeiramente com a visão do professor e posteriormente com a visão do aluno, apresentados abaixo, respectivamente.

Para que os alunos possam utilizar o Repositório é necessário que antes o professor da disciplina cadastre a turma a qual vai ministrar a aula, informando a turma, disciplina, conteúdo e um módulo do conteúdo. Também é necessário cadastrar usuário e senha para a turma, como apresenta a Figura 6. Caso o professor já tenha cadastrado uma turma, este necessita apenas editar o conteúdo para a aula.

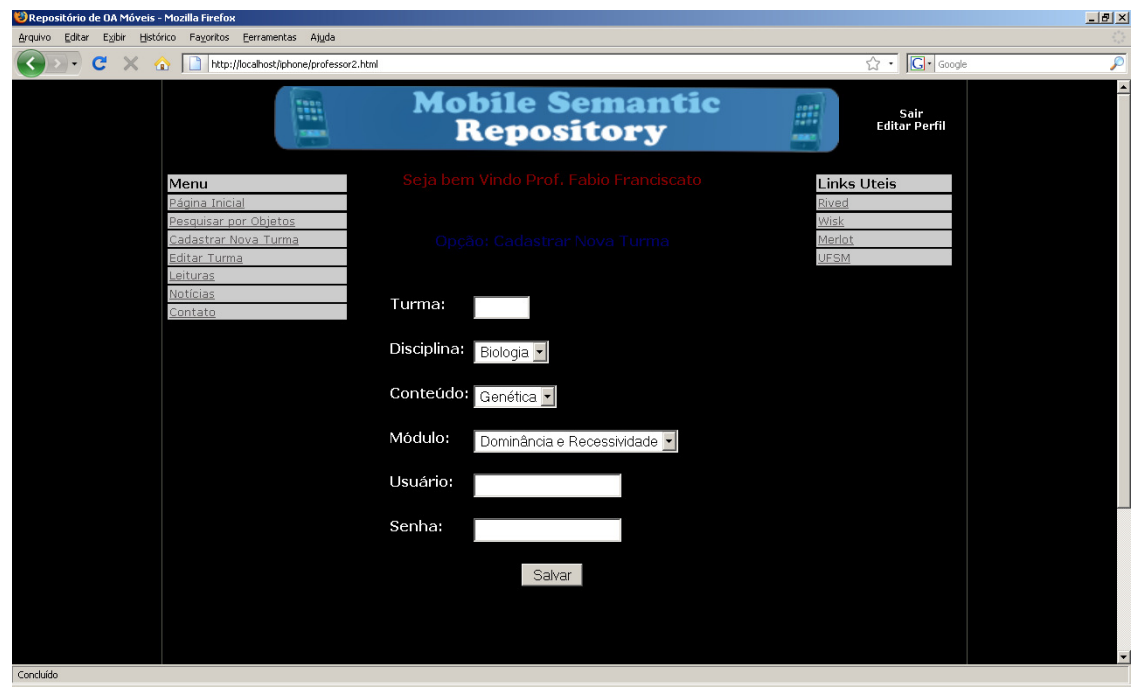

Figura 6. Tela de cadastro de turma na visão do professor

Com a turma e os devidos dados cadastrados, os alunos poderão fazer uso do repositório, confirmando nome de usuário e senha, informados pelo professor.

No caso do acesso dos alunos, o Repositório foi testado com dois Smartphones de diferentes fabricantes, um BlackBerry e um Iphone. Na Figura 7, são apresentadas três telas do Repositório, as quais foram acessadas pelos dispositivos móveis citados.
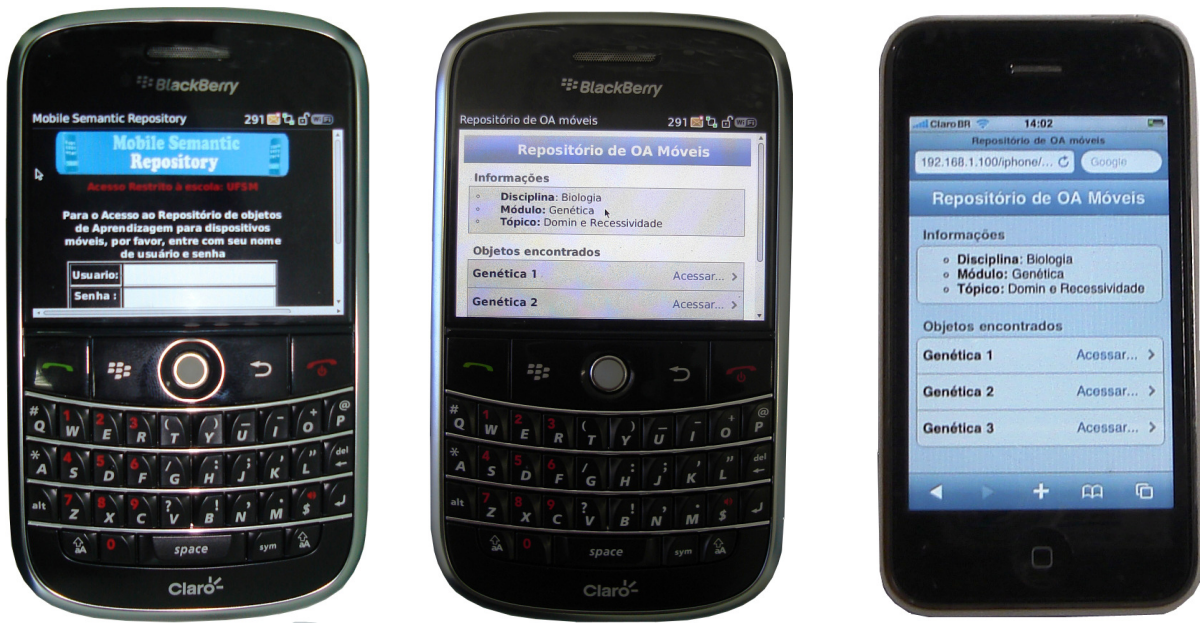

Figura 7. Fotos expondo algumas telas do Repositório acessadas pelo BlackBerry e Iphone respectivamente

As telas em questão apresentam, respectivamente, a tela de acesso ao Repositório e as telas da pagina principal do aluno, sendo que esta apresentará o nome 
da disciplina, o módulo e tópico que serão vistos em aula e os OA refinados pelo conteúdo e pelas configurações do dispositivo móvel do aluno. Selecionando um dos OA, este será executado pelo dispositivo móvel.

\section{Considerações Finais}

Muito tem se desenvolvido no cenário computacional para ajudar no processo de ensino/aprendizagem dos alunos, mas pouco tem se aproveitado. Objetos de Aprendizagem são recursos pedagógicos que podem facilitar o processo de aquisição do conhecimento pelos alunos, mas devido a vários problemas, como os abordados neste artigo, professores não estão fazendo uso desses materiais.

Foi para mudar este cenário que este artigo apresentou uma proposta de um repositório semântico de Objetos de Aprendizagem que pudesse ser utilizado em sala de aula pelos alunos, através de seus dispositivos móveis.

A escola não terá custos significativos para adotar as tecnologias necessárias e para o aluno será necessário que, no mínimo, seu dispositivo móvel tenha acesso à rede sem fio. Porém, as facilidades que a tecnologia traz não são determinantes para a utilização de Objetos de Aprendizagem em sala de aula, é necessário apoio e incentivo dos professores sobre a utilização dos OA e o comprometimento do aluno com essa nova abordagem no ensino.

Para trabalhos futuros pretende-se aperfeiçoar o repositório proposto e validá-lo em uma sala de aula presencial.

\section{Referências Bibliográficas}

ARAUJO, Moysés; Ferreira, M, A, Construindo uma Ontologia para pesquisa de Materiais e Objetos de Aprendizagem baseado na Web Semântica. Simpósio Brasileiro de Informática na Educação, SBIE. 2004

ARAUJO, Moysés. Educação a Distância e a Web Semântica: Modelagem Ontológica de Materiais e Objetos de Aprendizagem para a Plataforma CoL. Tese em Engenharia da Computação. USP. 2003

AUGUSTIN, I; et al. ISAM, joining context-awareness and mobility to building pervasive applications. Mobile Computing Handbook. Ed. Florida. 2004

BIZZARRO, S. Danilo. Aplicação de RDF para metadados de banco de dados de imagens de documentos. Londrina. 2006

CASTILlO, Sergio; Ayala, Geraldo. ARMOLEO: An architecture for Mobile Learning Objects. Universidad de Las Americas $18^{\text {th }}$ International Conference on Electronics, Communications and Computers. Mexico. 2008

CRUZ, L. J. C., Nicoleit, E. R., Giacomazzo, G. F., Zanette, E. N., Gonçalves, L. L. Objeto de Aprendizagem de Suporte ao Conteúdo Matemático de Limites para Dispositivos Móveis Baseado no Padrão SCORM - OALM. Hífen (Uruguaiana). , v.32, p.237 - 244, 2008.

FERREIRA, E, C, H. G. Geração Automática de Metadados: uma Contribuição para a Web Semântica. Tese de Engenharia. Universidade de São Paulo. 2006

FRANCISCATO, Fabio; Medina, R; Mozzaquatro, P; Ribeiro, P. Ontology for A Semantic Learning Objects Repository for Mobile Devices. IADIS www/internet 2009 
FRANCISCATO, Fabio; Medina, Roseclea. M-Learning e Android: um novo paradigma? Revista Novas Tecnologias na Educação - CINTED-UFRGS 2008

GUIMARÃES, F, J, Z. Utilização de ontologias no domínio B2C. Dissertação Mestrado Informática. RJ. 2002

HENDLER, J.; Berners-Lee, T.; Miller, E. Integrating Applications on the Semantic Web. Journal of the Institute of Electrical Engineers of Japan. v. 122 n.10. 2002

MERLOT. Multimidia Educational Resource for Learning and Online Teaching. Disponível em http://www.merlot.org/merlot/index.htm 1997

NETO, W, C, B. Web Semântica na Construção de Sistemas de Aprendizagem Adaptativos. Tese da Universidade Federal de Santa Catarina. Ciência da Computação. 2006

TAVARES, R; Rodrigues, G; Andrade, M; Santos, J; Cabral, L; Cruz, H; Monteiro, B; Gouveia, T; Picado, K; Objetos de Aprendizagem: Uma proposta de Avaliação da Aprendizagem Significativa. Livro Rived 2007

OLIVEIRA, L; Medina, R. Desenvolvimento de objetos de aprendizagem para dispositivos móveis: uma nova abordagem que contribui para a educação. Revista Novas Tecnologias na Educação.

OWL Web Ontology Language (OWL). Disponível em <http://www.w3.org/2004/OWL/> 2007

PROTÉGÉ. Protégé. Disponível em < http://protege.stanford.edu/> 1997

RAP. RDF API for PHP. Disponivel em <http://www.seasr.org/wpcontent/plugins/meandre/rdfapi-php/doc/index.html>. 2009

RIVED. Rede Interativa Virtual de Educação. Disponível em http://rived.mec.gov.br/ 2004

SIAU K, ; Nah F. H. Mobile Technology in Education. University of NebraskaLincoln 2008

SPARQL. SPARQL Query Language for RDF. Disponível em http://www.w3.org/TR/rdf-sparql-query/ 2008

STARR, S. Application of Mobile Technology in Learning \& Teaching: 'Mlearning'. Learning \& Teaching Enhancement Unit (LTEU). 2007

SCHLEMMER, E.; Zanela. S. A.; Barbosa, J.; Reinhard, N. M-Learning ou Aprendizagem com Mobilidade: Casos no contexto Brasileiro. 2007

TOLEDANO M. C. M. Learning objects for mobile devices: A case study in the Actuarial Sciences degree. Current Developments in technology-Assisted Education 2006

WILEY, D. Connecting learning objects to instructional design theory: a definition, a metaphor, and a taxonomy. The Instructional Use of Learning Objects. 2002

W3C. World Wide Web Consortion. Disponível em <http://www.w3.org/>. 2001 